\title{
Autopilot-based Nonlinear UAV Formation Controller with Extremum-Seeking
}

\author{
Feng Xie, Ximing Zhang, Rafael Fierro, and Mark Motter
}

\begin{abstract}
In this paper, we consider the problem of designing nonlinear robust formation controllers on a team of Unmanned Aerial Vehicles (UAV) using off-the-shelf autopilots. Two nonlinear robust formation control algorithms are presented. The first controller requires knowledge of the leader's velocity and acceleration. The second controller, on the other hand, does not have such a requirement. Additionally, the desired trajectory for the follower UAV is generated by a perturbation-based extremum-seeking (ES) controller. With these algorithms, two formation flight objectives have been achieved: (i) a reduction of communication overhead, and (ii) a maximum energy saving mission flight. Numerical simulations validate the performance of the proposed control methodology.
\end{abstract}

\section{INTRODUCTION}

Due to recent developments in electronics and computing, it is now possible to find small size, light weight, powerful embedded computers, and off-the-shelf ad-hoc wireless network devices on the market. As a result, by exploiting current technology, one can build a group of relatively small UAVs each having satisfactory capabilities within a reasonable budget. In adversary and dangerous missions, it might be convenient to have multiple small UAVs operating cooperatively. This leads to cost-efficiency, fault tolerance and robustness. For tasks such as obtaining sensory measurements over a wide area (e.g., forest fire monitoring [1]), multiple UAVs are desirable because they can accomplish the task more efficiently than a single UAV. The reader is referred to [2] where a survey of UAV cooperative control is provided.

Teams of UAVs flying in formations with precisely defined geometries lead to many advantages, such as energy saving when the vortex forces are taken into account [3]. In addition, formation control allows for intelligent leaders and single agent planning while followers can focus on other tasks. Leader-following is a common approach to build formations of multi-vehicle systems. The challenge here lies in designing a formation controller that is computationally simple, yet robust. In [4] a framework for controlling a group of UAVs is developed. The controller design utilizes input/output dynamic linearization techniques based on a model that included the induced rolling moment generated by the lead aircraft over the wing aircraft. A testbed for autonomous

F. Xie, X. Zhang, and R. Fierro are with the MARHES Lab, School of Electrical and Computer Engineering, Oklahoma State University, Stillwater, OK 74078, USA ffeng.xie, tony.zhang, rafael.fierro\}@okstate.edu

M. A. Motter is with the NASA Langley Research Center, Electronics System Branch, Hampton, VA 23681, USA m.a.motteralarc.nasa.gov flying controls is described in [5]. Authors in [6] develop an interesting experimental testbed for investigating close formation flight. In [7] a sliding-mode formation controller is proposed. Another relevant problem in multi-UAV systems that has received considerable attention is to design protocols such that a group of vehicles can achieve consensus upon certain variables of interest. Consensus problems in networks of agents have been addressed in [8].

Motivated by the idea of seeking the maximum induced lift for the follower UAV, some authors have proposed extremum-seeking (ES) formation flight controllers [9]. In this paper, we propose a multi-layer control architecture for practical and robust formation control and ES based on a cost effective autopilot ${ }^{1}$. In this scenario, the control objectives of the higher-level extremum-seeking controller are to locate the maximum induced lift points and to generate the desired trajectory for the middle-layer formation controller. To generate the desired trajectory, a perturbation-based algorithm is utilized to seek the maximum induced lift location while ensuring the trajectory is sufficiently smooth. It is assumed that the induced lift on the follower UAV is measurable or observable during the flight. The formation controller in turn generates velocity profiles to maintain a given formation geometry. Finally, the lower-level autopilot regulates the attitude of the follower UAV, maintains a desired height, and ensures that the UAV tracks the desired velocity profiles during the mission flight.

The remaining paper is organized as follows. Section II presents notation and mathematical preliminaries. Section III describes two different robust nonlinear formation controllers. Section IV is devoted to the extremum-seeking controller design. Simulation results are presented in Section V. Finally, concluding remarks are given in Section VI.

\section{NOTATION AND PRELIMINARIES}

We consider a group of $N$ unmanned aerial vehicles flying in formation. Sophisticated aircraft aerodynamical models can be found in many references, see for instance [10]. In this work, we simplify our analysis to an ideal scenario that the UAVs are flying in a wings-level steady-state and that the angle of attack and sideslip are considerably small, such that they can be ignored.

Usually, an off-the-shelf autopilot can provide two basic operational modes: (i) waypoint tracking mode, and (ii) holding mode. In the former, a sequence of points of interest can be uploaded into the autopilot before the mission is executed

\footnotetext{
${ }^{1}$ A product of Cloud Cap Technology, Inc.
} 
or during the flight. The autopilot then generates a path from the current position to the next point of interest and provides the control commands to the aircraft. In this mode, however, the user cannot precisely control the aircraft's position in the middle of two successive waypoints. In contrast, in holding mode, usually three hold channels are provided as follows: (1) the Mach hold, (2) the heading turn rate hold, and (3) the altitude hold. The autopilot continuously executes control commands sent to these hold channels. Although, some successful waypoint autopilot-based formation flight experiments have been reported in the literature [11], it would be more convenient to use the autopilot in holding mode when close formation flight is required. For instance, extremum-seeking, navigation in urban environments, and others require precise close formation flight. In this paper, we investigate the feasibility of using an off-the-shelf autopilot [12] in holding mode for the follower UAVs. The lead aircraft, on the other hand, has its autopilot set in waypoint tracking mode.

Based on the autopilot running in the holding mode, the lateral and longitudinal movements are decoupled. We can write a simplified model of the aircraft as [13]:

$$
\begin{aligned}
& {\left[\begin{array}{c}
\dot{x}_{i} \\
\dot{y}_{i} \\
\dot{\psi}_{i}
\end{array}\right]=\left[\begin{array}{cc}
\cos \psi_{i} & 0 \\
\sin \psi_{i} & 0 \\
0 & 1
\end{array}\right]\left[\begin{array}{c}
V_{i} \\
\omega_{i}
\end{array}\right],} \\
& \dot{V}_{i}=-\frac{1}{\tau_{v}} V_{i}+\frac{1}{\tau_{v}} V_{i c} \\
& \dot{\omega}_{i}=-\frac{1}{\tau_{\omega}} \omega_{i}+\frac{1}{\tau_{\omega}} \omega_{i c} \\
& \ddot{h_{i}}=-\frac{1}{\tau_{h_{a}}} \dot{h}_{i}-\frac{1}{\tau_{h_{b}}} h_{i}+\frac{1}{\tau_{h_{b}}} h_{i c}
\end{aligned}
$$

where the subscript $i \in I$ indicates the state variables of the $i^{\text {th }} \mathrm{UAV}, x_{i}$ and $y_{i}$ represent the positions in Cartesian coordinates, $V_{i}$ is the velocity, $\psi_{i}$ is the heading angle, and $h_{i}$ is the altitude. $V_{i c}, \omega_{i c}$, and $h_{i c}$ are the commands to the Mach hold, heading-turn-rate hold, and altitude hold channels of the autopilot, respectively. $\tau_{v}, \tau_{\omega}, \tau_{h_{a}}$, and $\tau_{h_{b}}$ are known positive constants that depend on the autopilot.

\section{LEADER-FOLLOWING FORMATION CONTROL}

In this section, we set up a kinematic model for an UAV formation flight system. Assuming that the UAVs are flying at a constant altitude, we can consider an operator specified the $i^{t h} \mathrm{UAV}$ motion $a_{i}(t) \in \mathbb{R}^{2}, i \in\{1,2, \ldots, N\}$ given as follows

$$
a_{i}(t)=x_{i}(t) \hat{\imath}+y_{i}(t) \hat{\jmath}
$$

where $x_{i}(t)$ and $y_{i}(t) \in \mathbb{R}$ represent the respective Cartesian coordinates in an earth fixed reference inertial frame $\mathcal{I}$. Let $F_{j k}^{d}(t) \in \mathbb{R}^{2}$ be a desired formation that allows a wing airplane $k$ to follow a leader airplane $j$ given by

$$
F_{j k}^{d}(t)=\left[\begin{array}{ll}
l_{j k}^{d} & \eta_{j k}^{d}
\end{array}\right]^{T},
$$

where $l_{j k}^{d}(t) \in \mathbb{R}_{+}$is the desired distance and $\eta_{j k}^{d}(t) \in$ $\left[\frac{\pi}{2}, \frac{3 \pi}{2}\right]$ is the desired relative bearing angle as shown in
Figure 1 . Consequently by changing $F_{j k}^{d}(t)$ we are able to define different formation shapes. Then the actual formation for a wing airplane $k$ to follow a leader airplane $j$ is described by

$$
F_{j k}(t)=\left[\begin{array}{ll}
l_{j k} & \eta_{j k}
\end{array}\right]^{T} \in \mathbb{R} \times\left[\frac{\pi}{2}, \frac{3 \pi}{2}\right],
$$

in which the relative distance is defined as

$$
l_{j k}(t)=\left\|a_{j}-a_{k}\right\|_{2},
$$

where $\|\cdot\|_{2}$ denotes the standard Euclidean norm; $a_{j}(t)=$ $x_{j}(t) \hat{\imath}+y_{j}(t) \hat{\jmath}$ and $a_{k}(t)=x_{k}(t) \hat{\imath}+y_{k}(t) \hat{\jmath}$ are the specified operators of the leader airplane and the wing airplane in $\mathcal{I}$; and $j, k$ stand for leader and wing, respectively. The relative bearing is given by

$$
\eta_{j k}(t)=\pi+\zeta-\psi_{j}
$$

where $\zeta(t)=\arctan 2\left(y_{j}-y_{k}, x_{j}-x_{k}\right)$.

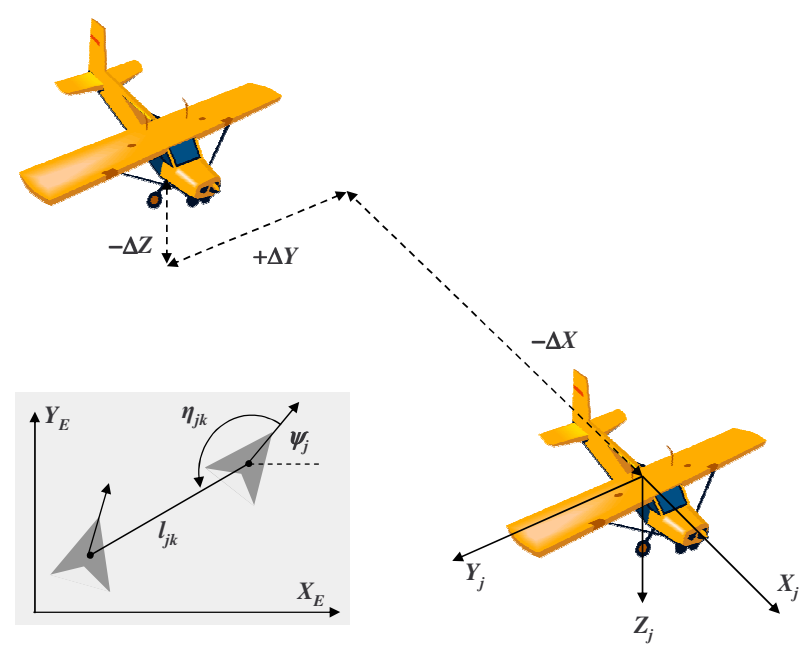

Fig. 1. Wing-leader configuration.

Taking the time derivative of equation (5), we have

$$
\dot{F}_{j k}=\left[\begin{array}{c}
i_{j k} \\
\dot{\eta}_{j k}
\end{array}\right]=\left[\begin{array}{c}
V_{k} \cos \gamma_{j k}-V_{j} \cos \eta_{j k} \\
\frac{1}{l_{j k}}\left(V_{j} \sin \eta_{j k}-V_{k} \sin \gamma_{j k}-l_{j k} \omega_{j}\right)
\end{array}\right] .
$$

Rearranging (8), we can derive the following nonlinear kinematic model for the leader-following formation flight system

$$
\dot{F}_{j k}(t)=g_{1}(\cdot) V_{k}+g_{2}(\cdot) V_{j}+g_{3}(\cdot) \omega_{j},
$$

where the vector fields $g_{1}(\cdot), g_{2}(\cdot)$, and $g_{3}(\cdot) \in \mathbb{R}^{2}$ are defined as $g_{1}(\cdot)=\left[\begin{array}{l}\cos \gamma_{j k} \\ -\frac{1}{l_{j k}} \sin \gamma_{j k}\end{array}\right], g_{2}(\cdot)=\left[\begin{array}{c}-\cos \eta_{j k} \\ \frac{1}{l_{j k}} \sin \eta_{j k}\end{array}\right]$, $g_{3}(\cdot)=\left[\begin{array}{l}0 \\ -1\end{array}\right]$, with $\gamma_{j k}(t) \triangleq \psi_{j}+\eta_{j k}-\psi_{k} . V_{k}(t)$ is the linear velocity of the follower. $V_{j}(t)$ and $\omega_{j}(t)$ are the linear and angular velocities of the leader. Our control objective is to design the formation controller $\left(V_{k}(t), \omega_{k}(t)\right)$ to drive the 
wing UAV to track a desired formation $F_{j k}^{d}(t)$. To this end, we define the formation error as

$$
e=F_{j k}^{d}(t)-F_{j k}(t),
$$

where $e(t) \in \mathbb{R}^{2}$. Taking the derivative of (10) with respect to time and combining with (9), the following is obtained

$$
\dot{e}=\dot{F}_{j k}^{d}-g_{1}(\cdot) V_{k}-g_{2}(\cdot) V_{j}-g_{3}(\cdot) \omega_{j} .
$$

It is obvious that the input matrix $g_{1}(\cdot)$ of the error system (11) is not invertible. Thus we cannot design the control based on (11). To facilitate the control design on this system, we first use the dynamic extension method from [14] to render (11) into a relative degree system. Differentiating both sides of (11) with respect to time and after some algebraic and trigonometric manipulation, we obtain

$$
\begin{aligned}
\ddot{e}= & \ddot{F}_{j k}^{d}-\left[\frac{\partial g_{1}(\cdot)}{\partial \gamma_{j k}} \dot{\eta}_{j k}+\frac{\partial g_{1}(\cdot)}{\partial l_{j k}} \dot{l}_{j k}\right] V_{k}+g(\cdot) u_{k} \\
& +f_{1}(\cdot) \varpi_{j}+f_{2}(\cdot) \dot{\varpi}_{j} .
\end{aligned}
$$

The new input matrix is given by

$$
\begin{aligned}
g(\cdot) & =\left[\begin{array}{ll}
-g_{1}(\cdot) & \frac{\partial g_{1}(\cdot)}{\partial \gamma_{j k}} V_{k}
\end{array}\right] \\
& =\left[\begin{array}{cc}
-\cos \gamma_{j k} & -V_{k} \sin \gamma_{j k} \\
\frac{1}{l_{j k}} \sin \gamma_{j k} & -\frac{V_{k}}{l_{j k}} \cos \gamma_{j k}
\end{array}\right] \in \mathbb{R}^{2 \times 2},
\end{aligned}
$$

and the new matrices are

$$
\begin{aligned}
& f_{1}(\cdot)=\left[\begin{array}{ll}
-\dot{g}_{2}(\cdot) & -\frac{\partial g_{1}(\cdot)}{\partial \gamma_{j k}} V_{k}
\end{array}\right] \in \mathbb{R}^{2 \times 2}, \\
& f_{2}(\cdot)=\left[\begin{array}{ll}
-g_{2}(\cdot) & -g_{3}(\cdot)
\end{array}\right] \in \mathbb{R}^{2 \times 2} .
\end{aligned}
$$

Also, we define the leader linear and angular velocity vector as $\varpi_{j}=\left[\begin{array}{ll}V_{j} & \omega_{j}\end{array}\right]^{T} \in \mathbb{R}^{2}$, and the follower new control input as $u_{k}(t) \triangleq\left[\begin{array}{ll}\dot{V}_{k} & \omega_{k}\end{array}\right]^{T} \in \mathbb{R}^{2}$. It is not difficult to check that the new input matrix $g(\cdot)$ is nonsingular under the condition that the flight speed $V_{k} \geq V_{\min }>0$ and $l_{j k} \geq$ $l_{\text {min }}>0 . V_{\text {min }}$ is the UAV stalling speed, and $l_{\text {min }}$ is the minimum distance to avoid collision between the two UAVs.

\section{A. A Primary Nonlinear Approach}

As a primary approach in this sub-section, we assume the leader linear and angular velocity and acceleration vectors $\varpi_{j}$ and $\dot{\varpi}_{j}$ are known to the follower aircraft (by leader's onboard instruments and the communication link between leader and follower). We first design the following filtered signal $r(t)=\left[r_{1} r_{2}\right]^{T} \in \mathbb{R}^{2}$

$$
r(t)=\dot{e}+K e,
$$

where $K=\left[\begin{array}{cc}k_{1} & 0 \\ 0 & k_{2}\end{array}\right]$, and $k_{1,2} \in \mathbb{R}_{+}$are design gain constants. Differentiating (13) with respect to time and substituting in (12) produces

$$
\begin{aligned}
\dot{r}(t)= & \ddot{F}_{j k}^{d}-\left(\frac{\partial g_{1}(\cdot)}{\partial \gamma_{j k}} \dot{\eta}_{j k}+\frac{\partial g_{1}(\cdot)}{\partial l_{j k}} \dot{l}_{j k}\right) V_{k}+g(\cdot) u_{k} \\
& +f_{1}(\cdot) \varpi_{j}+f_{2}(\cdot) \dot{\varpi}_{j}+K \dot{e} .
\end{aligned}
$$

Based on a Lyapunov analysis, the formation control law becomes

$$
\begin{aligned}
u_{k}= & g^{-1}(\cdot)\left[-\ddot{F}_{j k}^{d}+\left(\frac{\partial g_{1}(\cdot)}{\partial \gamma_{j k}} \dot{\eta}_{j k}+\frac{\partial g_{1}(\cdot)}{\partial l_{j k}} i_{j k}\right) V_{k}\right. \\
& \left.-f_{1}(\cdot) \varpi_{j}-f_{2}(\cdot) \dot{\varpi}_{j}-K \dot{e}-\phi(r)\right],
\end{aligned}
$$

where $\phi(r) \in \mathbb{R}^{2}$ is a sliding vector function so that the sliding condition is guaranteed.

Theorem 3.1: The control law of (15) ensures stable sliding surface dynamics of the system in (13) and that all system signals are bounded under closed-loop operation and the tracking error is asymptotically stable in the sense that $\lim _{t \rightarrow \infty} e(t), \dot{e}(t)=0$.

Remark 3.2: A generic sliding vector function $\phi(r)$ is given in the above control design, so a variety of available sliding vector functions can be substituted in to reduce the chattering and to achieve satisfactory performance.

\section{B. A Secondary Nonlinear Approach}

The effectiveness of the control law introduced in the previous section is based on the assumption, the leader's position, attitude, velocity and acceleration information are all known to the follower. Those data can be obtained by onboard instruments (on leader and/or follower) and intervehicle communications. This is a common assumption in the formation flight control literature. However, in reality, due to the payload limitation or communication failure (for example, under electronic countermeasures), this assumption might not always hold. Consequently, control laws that assume full knowledge of the lead aircraft state cannot guarantee a desired formation in the presence of communication failures. Some issues regarding control design under limited communication are addressed in [15].

In this section, we present a robust nonlinear formation controller design that does not assume the leader's velocity and acceleration are known. Specifically, by expanding the second term on the right side of equation (14) along with equation (8), we can rearrange (14) into

$$
\begin{aligned}
\dot{r}(t)= & \ddot{F}_{j k}^{d}-\left[\begin{array}{l}
\frac{1}{l_{j k}} \sin ^{2} \gamma_{j k} \\
\frac{2}{l_{j k}^{2}} \cos \gamma_{j k} \sin \gamma_{j k}
\end{array}\right] V_{k}^{2}+g(\cdot) u_{k} \\
& +f_{1}(\cdot) \varpi_{j}+f_{2}(\cdot) \dot{\varpi}_{j}+f_{3}(\cdot) \varpi_{j} V_{j}+K \dot{e},(16)
\end{aligned}
$$

where $f_{1}(\cdot), f_{2}(\cdot)$, and $f_{3}(\cdot)$ are functions of $F_{j k}(t), V_{k}(t)$, and $\gamma_{j k}(t)$. Note that $f_{1}(\cdot), f_{2}(\cdot)$, and $f_{3}(\cdot)$ are bounded since $l_{j k} \geq l_{\text {min }}>0$ and $V_{k}(t) \in \mathcal{L}_{\infty}$.

We make the following assumptions:

Assumption 3.3: During the formation flight, the leader UAV is stably tracking some desired trajectories $\varpi_{j}^{d}=$ $\left[V_{j}^{d} \omega_{j}^{d}\right]^{T}, \dot{\varpi}_{j}^{d}=\left[\dot{V}_{j}^{d} \dot{\omega}_{j}^{d}\right]^{T} \in \mathbb{R}^{2}$ with $\varpi_{j}^{d}, \dot{\varpi}_{j}^{d}, \ddot{\varpi}_{j}^{d} \in \mathcal{L}_{\infty}$ so that we can assume $\varpi_{j}, \dot{\varpi}_{j}, \ddot{\varpi}_{j} \in \mathcal{L}_{\infty}$.

Assumption 3.4: All other terms in (16) except $\varpi_{j}$ and $\dot{\varpi}_{j}$ are known.

Remark 3.5: Using equation (3), it is possible to generate a leader UAV trajectory so that Assumption 3.3 holds. 
The follower control vector $u_{k}(t)$ in (14) becomes

$$
\begin{aligned}
u_{k}= & g^{-1}(\cdot)\left(-\ddot{F}_{j k}^{d}+\left[\begin{array}{c}
\frac{1}{l_{j k}} \sin ^{2} \gamma_{j k} \\
\frac{2}{l_{j k}^{2}} \cos \gamma_{j k} \sin \gamma_{j k}
\end{array}\right] V_{k}^{2}\right. \\
& \left.-k_{s} e-\beta_{1} \operatorname{sgn}(e(t))\right)
\end{aligned}
$$

where $k_{s}$ and $\beta_{1}$ are constant positive control gains. After substituting $u_{k}$ into (16), the closed-loop system is given by

$$
\begin{aligned}
\dot{r}(t)= & f_{1}(\cdot) \varpi_{j}+f_{2}(\cdot) \dot{\varpi}_{j}+f_{3}(\cdot) \varpi_{j} V_{j}+K \dot{e} \\
& -k_{s} e-\beta_{1} \operatorname{sgn}(e(t)) .
\end{aligned}
$$

Before stating the main result of this section, we give the following lemma which will be invoked later.

Lemma 3.6: Let the auxiliary function $\Gamma(t) \in \mathbb{R}$ be defined as follows

$\Gamma \triangleq r^{T}\left[f_{1}(\cdot) \varpi_{j}+f_{2}(\cdot) \dot{u}_{j}+f_{3}(\cdot) \varpi_{j} V_{j}+K \dot{e}-\beta_{1} \operatorname{sgn}(e)\right]$

If the control gain $\beta_{1}$ is selected to satisfy the sufficient condition

$$
\begin{aligned}
\beta_{1} \quad> & \left\|f_{1}(\cdot) \varpi_{j}+f_{2}(\cdot) \dot{\varpi}_{j}+f_{3}(\cdot) \varpi_{j} V_{j}+K \dot{e}\right\|_{2}+ \\
& \frac{1}{\|K\|_{2}}\left\|\frac{d\left(f_{1}(\cdot) \varpi_{j}+f_{2}(\cdot) \dot{\varpi}_{j}+f_{3}(\cdot) \varpi_{j} V_{j}+K \dot{e}\right)}{d t}\right\|_{2}
\end{aligned}
$$

where $K$ is defined in (13), then

$$
\int_{t_{0}}^{t} \Gamma(\tau) d \tau \leqslant \zeta_{b}
$$

where the positive constant $\zeta_{b} \in \mathbb{R}$ is defined as

$$
\begin{aligned}
\zeta_{b} \triangleq & \beta_{1}\left\|e\left(t_{0}\right)\right\|_{1}-e^{T}\left(t_{0}\right)\left[f_{1}\left(t_{0}\right) \varpi_{j}\left(t_{0}\right)+f_{2}\left(t_{0}\right) \dot{u}_{j}\left(t_{0}\right)\right. \\
& \left.+f_{3}\left(t_{0}\right) \varpi_{j}\left(t_{0}\right) V_{j}\left(t_{0}\right)+K \dot{e}\left(t_{0}\right)\right]
\end{aligned}
$$

where the notation $\|\cdot\|_{1}$ denotes the $\mathcal{L}_{1}$ norm.

We now state the main stability result for the second controller in the following theorem.

Theorem 3.7: The control law of (17) ensures that all system signals are bounded under closed-loop operation and the tracking error is asymptotically stable in the sense that $\lim _{t \rightarrow \infty} e(t), \dot{e}(t)=0$.

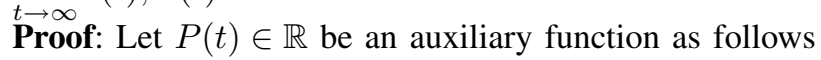

$$
P(t) \triangleq \zeta_{b}-\int_{t_{0}}^{t} \Gamma(\tau) d \tau \geqslant 0,
$$

where $\zeta_{b}$ and $\Gamma(t)$ have been defined in Lemma 3.6. Based on the non-negativity of $P(t)$, we define a Lyapunov function candidate $V(t)$ by

$$
V \triangleq \frac{1}{2} r^{T} r+\frac{1}{2} k_{s} e^{T} e+P .
$$

After taking the time derivative of (23), we have $\dot{V}=r^{T} \dot{r}+$ $k_{s} e^{T} \dot{e}+\dot{P}$. Then by utilizing the closed-loop dynamics of (18) and (22), we can obtain the following expression for $\dot{V}(t)$

$$
\begin{aligned}
\dot{V}= & r^{T}\left[f_{1}(\cdot) \varpi_{j}+f_{2}(\cdot) \dot{\varpi}_{j}+f_{3}(\cdot) \varpi_{j} V_{j}+K \dot{e}\right. \\
& \left.-k_{s} e-\beta_{1} \operatorname{sgn}(e(t))\right]+k_{s} e^{T} \dot{e}-\Gamma .
\end{aligned}
$$

Rearranging the first term on the right hand side of (24) and using the definition of $\Gamma$, we get

$$
\dot{V}=-k_{s} r^{T} e+k_{s} e^{T} \dot{e}=-K k_{s} e^{T} e \leqslant-k_{m i n} k_{s}\|e\|^{2}
$$

where $k_{\min }=\min \left\{k_{1}, k_{2}\right\} . e(t) \in \mathcal{L}_{\infty} \cap \mathcal{L}_{2}$ and $\lim _{t \rightarrow \infty} e(t)=$ 0. From Lemma A.8 of [16] and the definition of (13), one readily obtains $r(t) \in \mathcal{L}_{\infty} \cap \mathcal{L}_{2}$ and $\lim r(t)=0$. From (13) again, it is easy to see $\dot{e}(t) \in \mathcal{L}_{\infty} \cap \mathfrak{t \rightarrow \infty}_{2}$ and $\lim _{t \rightarrow \infty} \dot{e}(t)=0$.

\section{ES-BASED DESIRED TRAJECTORY GENERATION}

In UAV close formation flight, the follower UAV will ride on the upwash field of the wingtip vortex of the leader UAV. This will maximize the energy saved during flight. Furthermore, the Dryden flight showed that the lift force $L(t)$ received by the follower UAV in the wakes of the upwash is a function of the lateral wingspan separation $y(t)$ and the vertical wingspan separation $z(t)$ [3]. In fact, a maximum lift point does exist, and the induced lift $L(t)$ can be estimated [9]. Motivated by the Dryden flight results, our objective is to design an ES controller to seek the existing maximum lift point. Thus, the ES controller generates a reference trajectory for the follower UAV.

The robust formation controllers given in the previous section allow the follower UAV to track a desired trajectory. Following the work presented in [17] and the guidelines given in [18], the extremum-seeking can be decoupled into lateral and vertical control loops. The extremum-seeking algorithm in the lateral control loop produces the desired lateral separation trajectory, $y_{d}(t)$, as follows

$$
\begin{aligned}
& y_{d}(t)=\delta_{l t} \sin \left(\omega_{l t} t\right)+\hat{y}_{d}(t) \\
& \dot{\hat{y}}_{d}(t)=k_{l t}(L(t)-\vartheta(t)) \delta_{l t} \sin \left(\omega_{l t} t-\theta_{l t}\right) \\
& \dot{\vartheta}(t)=-\omega_{h l t} \vartheta(t)+\omega_{h l t} L(t)
\end{aligned}
$$

where $\delta_{l t} \in \mathbb{R}$ is a small positive constant and represents the perturbation amplitude, $\omega_{l t} \in \mathbb{R}$ is a positive constant and represents the perturbation frequency, $k_{l t}$ and $\omega_{h l t} \in \mathbb{R}$ are constant filter parameters to be designed, $\theta_{l t}$ is a constant phase delay, $\hat{y}_{d}(t)$, and $\vartheta(t)$ are filtered signals. The desired wingspan vertical separation, $z_{d}(t)$, is generated in a similar fashion. Figure 2 illustrates a block diagram of a multi-layer two degree of freedom ES formation flight controller.

\section{Simulation Results}

The performance of the proposed controllers have extensively been tested on simulation. Matlab/Simulink is used to simulate Piper J3Cub UAVs equipped with Piccolo autopilots. Chattering is always an issue in sliding-mode like control design. In order to minimize chattering, $\arctan (\cdot)$ is used instead of $\operatorname{sgn}(\cdot)$ in (15) and (17). To simplify notation, the control algorithms described in Sections III-A and III-B are referred as $C_{1}$ and $C_{2}$, respectively.

In the simulation scenario, the initial conditions of the leader are $x_{j}(0)=y_{j}(0)=0 \mathrm{~m}, z_{j}(0)=1000 \mathrm{~m}, V_{j}(0)=$ $17.5 \mathrm{~m} / \mathrm{s}$, and $\omega_{j}(0)=0 \mathrm{rad} / \mathrm{s}$. For the follower, the initial conditions are $x_{k}(0)=150 \mathrm{~m}, y_{k}(0)=20 \mathrm{~m}, z_{k}(0)=980$ 


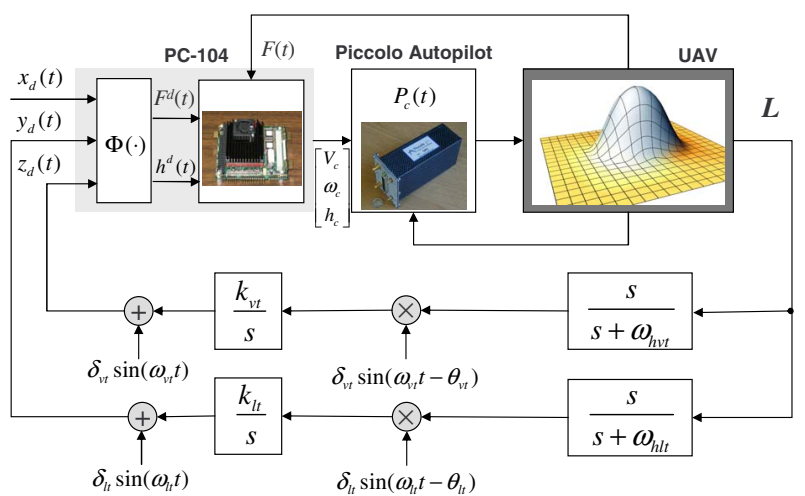

Fig. 2. Block diagram of extremum-seeking ES formation flight controller.

$\mathrm{m}, V_{k}=20 \mathrm{~m} / \mathrm{s}$, and $\omega_{k}(0)=0 \mathrm{rad} / \mathrm{s}$. The desired formation is $l_{j k}^{d}=100 \mathrm{~m}$ and $\eta_{j k}^{d}=\frac{5}{4} \pi \mathrm{rad}$.

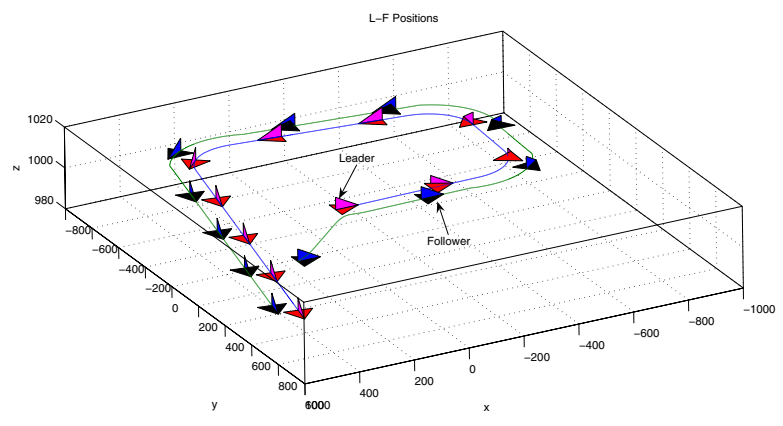

Fig. 3. Trajectories of the UAVs in close formation.

Since the trajectories of the wing-airplane under the two controllers are very similar, we only present in Figure 3, the trajectory of the 2-UAV team under the action of $C_{2}$. As it can be seen, the wing-airplane is able to maintain the desired relative distance and bearing angle with respect to the lead aircraft. Figures 4 and 5 depict the control inputs and formation error when $C_{1}$ is used.
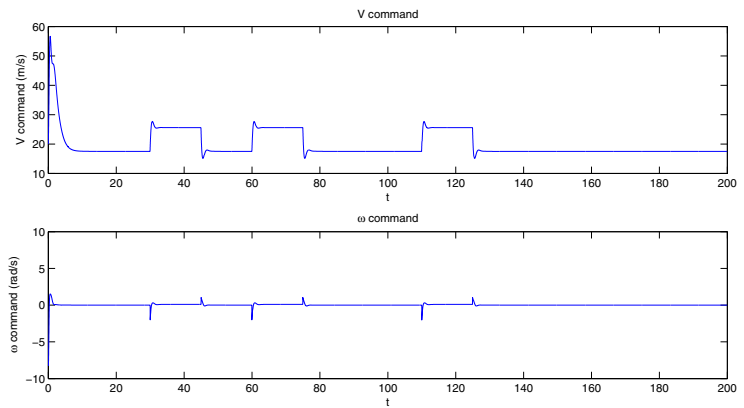

Fig. 4. Control inputs using $C_{1}$.

Furthermore, Figures 6 and 7 show the control inputs and formation error under $C_{2}$. Note that the initial amplitudes of the control inputs under $C_{2}$ are higher than the ones given by $C_{1}$. We anticipate this behavior since $C_{2}$ assumes no information about the velocity and acceleration of the leader
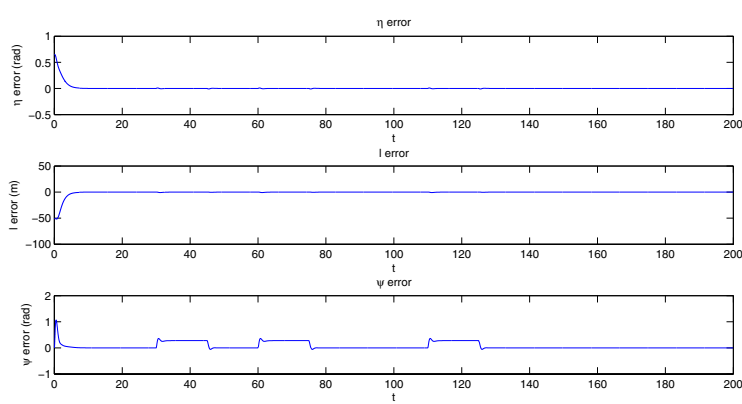

Fig. 5. Error under the action of $C_{1}$.

aircraft. Nevertheless, the overall performance of the closedloop system under $C_{2}$ is comparable with the performance under $C_{1} . C_{2}$ is the preferred controller in case of intervehicle communication failures or when the communication bandwidth is limited. A switching-logic scheme can be designed so that $C_{1}$ is used when communication between UAVs is possible and the follower UAV has access to its leader's velocity and acceleration; otherwise, $C_{2}$ should be switched in. Stability of the switching formation controller becomes an issue that would require further investigation. This analysis is out of the scope of this paper. We also
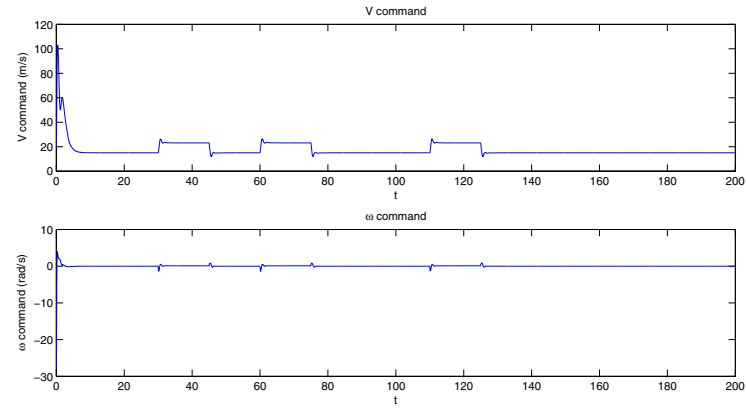

Fig. 6. Control inputs using $C_{2}$.
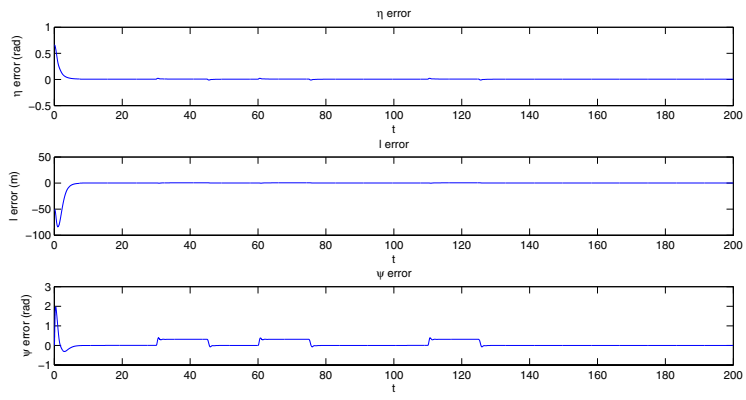

Fig. 7. Error under the action of $C_{2}$.

simulate the extremum-seeking ES higher-level controller outlined in Section IV. The experimental data of NASA Dryden flight research center [3] is used for this purpose. We follow the convention given in [3], that is the relative lateral 
position $Y$ is zero when the wingtips are aligned (refer to Figure 1). $Y$ becomes increasingly negative as the wingtip overlap increases. Similarly, the relative vertical position $Z$ is zero when the wingtips are aligned. $Z$ is negative, if the wing airplane is below the leader airplane. Finally, the longitudinal separation $X$ is fixed at a distance of $112 \mathrm{ft}$.

In the experiment, the peak values occur at $Y=-4.9 \mathrm{ft}$. (wings overlapped), and $Z=0.0 \mathrm{ft}$. Controller $C_{2}$ is used to track the reference trajectory generated by the ES controller. The initial initial lateral separation is $Y(0)=-11.3 \mathrm{ft}$. Both UAVs are flying at the same altitude, i.e., $Z=0 \mathrm{ft}$. The perturbation and filter parameters are $\delta_{l t}=0.12 \mathrm{ft}$., $\omega_{l t}=3$ $\mathrm{rad} / \mathrm{s}, k_{l t}=0.175, \theta_{l t}=0$, and $\omega_{h l t}=0.04 \mathrm{rad} / \mathrm{s}$.

The simulation results are presented in Figure 8 and 9. As it can be seen, the desired lateral position converges to $-4.9 \mathrm{ft}$ after $t=200 \mathrm{~s}$. Also, the induced lift asymptotically stabilizes at the the maximum point of about $L=750$.

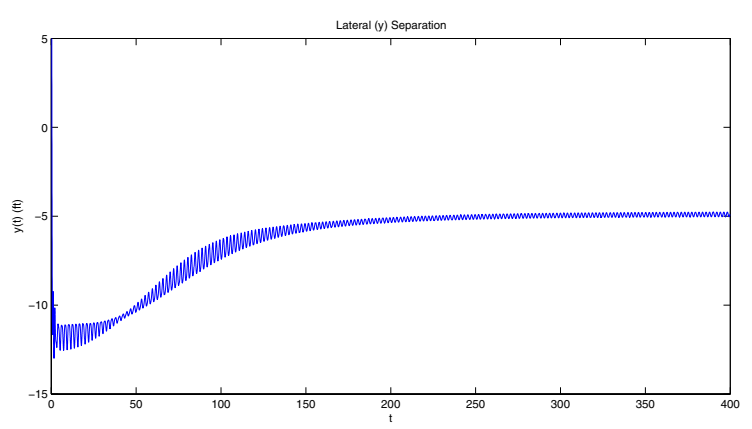

Fig. 8. UAV lateral separation.

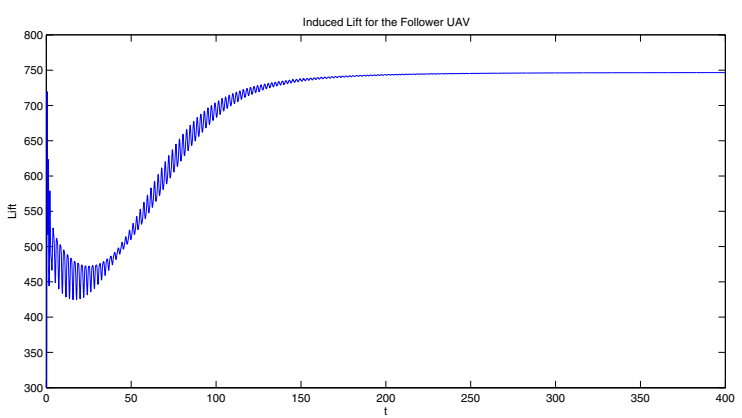

Fig. 9. Induced lift.

\section{CONCLUSIONS}

In this paper, we present a hierarchical control system that allows a team of UAVs to navigate in close formation. The lower-layer consists of off-the-shelf autopilots capable of trajectory generation in waypoint mode or holding mode. At the higher-layer, two robust, stable nonlinear controllers are developed and integrated with an extremum-seeking algorithm. This multi-layer control scheme allows a team of UAVs to perform complex navigation tasks with maximum energy saving under limited inter-vehicle communication.
Specifically, the second control law eliminates the requirement of leader's velocity and acceleration information, which significantly reduces the communication overhead.

Currently, these controllers are being implemented on an experimental testbed of terrestrial UAVs. Although, extremum-seeking cannot be implemented on terrestrial UAVs, we will be able to evaluate the performance and robustness of the controllers to communication failures and delays. This evaluation is critical before implementing the algorithms on actual unmanned aerial vehicles.

\section{ACKNOWLEDGEMENTS}

This work is supported in part by NSF grants \#0311460 and CAREER \#0348637 and by the U.S. Army Research Office under grant DAAD19-03-1-0142 (through the University of Oklahoma).

\section{REFERENCES}

[1] D. W. Casbeer, R. W. Beard, T. W. McLain, S.-M. Li, and R. K. Mehra, "Forest fire monitoring with multiple small UAVs," in Proc. American Control Conference, Portland, OR, June 2005, pp. 3530-3535.

[2] A. Ryan, M. Zennaro, A. Howell, R. Sengupta, and J. K. Hedrick, "An overview of emerging results in cooperative UAV control," in Proc. IEEE Conf. on Decision and Control, Atlantis, Paradise Island, Bahamas, December 14-17 2004, pp. 602-607.

[3] M. J. Vachon, R. J. Ray, K. R. Walsh, and K. Ennix, "F/A-18 performance benefits measured during the autonomous formation flight project," NASA Dryden Flight Research Center, Edwards, California., Tech. Rep. NASA/TM-2003-210734, 2003.

[4] R. Fierro, C. Belta, J. Desai, and V. Kumar, "On controlling aircraft formations," in Proc. IEEE Conf. on Decision and Control, Orlando, FL, December 2001, pp. 1065-1070.

[5] M. A. Motter, "Autonomous flying controls testbed," in AUVSI's Unmanned Systems North America, Baltimore MD, June 28-30 2005.

[6] J. M. Fowler and R. D'Andrea, "A formation flight experiment," in IEEE Control Systems Magazine, vol. 23, Oct 2003, pp. 35-43.

[7] S. N. Singh, M. Pachter, P. Chandler, S. Banda, S. Rasmussen, and C. Schumacher, "Input-output invertibility and sliding mode control for close formation flying of multiple uavs," Int. J. Robust Nonlinear Control, vol. 10, pp. 779-797, 2000.

[8] R. Olfati-Saber and R. M. Murray, "Consensus problems in networks of agents with switching topology and time-delays," IEEE Trans. on Automatic Control, vol. 49, no. 9, pp. 1520-1533, 2004.

[9] P. Binetti, K. B. Ariyur, and M. Krstic, "Control of formation flight via extremum seeking," in Proc. American Control Conference, Anchorage, AK, May 2002, pp. 2848-2853.

[10] B. L. Stevens and F. L. Lewis, Aircraft Control and Simulation, 2nd ed. Wiley, 2003.

[11] S. Bayraktar, G. E. Fainekos, and G. J. Pappas, "Experimental cooperative control of fixed-wing unmanned aerial vehicles," in Proc. IEEE Conf. on Decision and Control, Atlantis, Paradise Island, December 14-17 2004, pp. 4292-4298.

[12] B. Vaglienti, R. Hoag, and M. Niculescu, Piccolo System User's Guide, Cloud Cap Technology, Hood River, OR 97031, April 2005.

[13] M. Pachter, J. J. D'Azzo, and A. Proud, "Tight formation flight control," Guidance, Control, and Dynamics, vol. 24, no. 2, pp. 246254, 2001.

[14] A. Isidori, Nonlinear Control Systems, 3rd ed. London: SpringerVerlag, 2001.

[15] D. Hristu and K. Morgansen, "Limited communication control," Systems and Control Letters, vol. 37, pp. 193-205, July 1999.

[16] M. de Queiroz, D. Dawson, S. Nagarkatti, and F. Zhang, LyapunovBased Control of Mechanical Systems. Birkhaeuser, Boston: SpringerVerlag, 2000.

[17] K. B. Ariyur and M. Krstic, "Analysis and design of multivariable extremum seeking," in Proc. American Control Conference, Anchorage, AK, May 8-10 2002, pp. 2903-2908.

[18] M. A. Rotea, "Analysis of multivariable extremum seeking algorithms," in Proc. American Control Conference, Chicago, IL, June 2000, pp. 433-437. 